\title{
Extremely rare and unusual case of retroperitoneal and pelvic metastasis from squamous cell carcinoma of vallecula
}

\author{
Abhishek Purkayastha, Neelam Sharma, Virender Suhag \\ Department of Radiation Oncology, Army Hospital Research and Referral, Dhaula Kuan, New Delhi, India
}

Received December 24, 2015; Revised June 19, 2016; Accepted June 20, 2016; Published Online June 21, 2016

Case Report

\begin{abstract}
Carcinoma of oropharynx generally metastasizes to lungs, liver and bone while retroperitoneal and pelvic metastasis is very rare. We hereby report an extremely rare and unusual case of retroperitoneal and pelvic metastasis from squamous cell carcinoma of vallecula. To the best of our knowledge, this case is one of the scantly reported cases of this kind in the world. A 60-year-old male presented with complaints of dysphagia and hoarseness of voice of four months duration. Clinical evaluation and computed tomography scan of face and neck showed growth right vallecula and a biopsy revealed squamous cell carcinoma. Metastatic work up was negative. We treated him with definitive chemo-radiation, which he tolerated well. During follow up, he presented with dyspepsia, abdominal discomfort and weight loss. Whole body positron emission tomography scan revealed disseminated retroperitoneal and pelvic lymph node deposits, which were confirmed as metastasis of squamous cell carcinoma on image guided fine needle aspiration cytology. Patient was exhibited palliative chemotherapy in view of extensive metastasis. However, his general condition deteriorated and he finally succumbed to his illness. The aim of this case is to highlight the extreme rarity, the diagnostic and therapeutic challenges and the overall dismal prognosis of this condition.
\end{abstract}

Keywords: Metastasis; Pelvic lymph nodes; Retroperitoneum; Squamous cell carcinoma; Vallecula

\section{Introduction}

Distant metastatic spread from oropharyngeal cancer or any head and neck malignancy is relatively uncommon. It affects approximately $15 \%$ of all patients during the course of disease. ${ }^{1}$ The most common sites for distant metastasis are lung, bone, and liver ${ }^{1}$ while distant metastasis without local recurrence is much rarer. $^{2}$ Carcinoma oropharynx metastasizing to retroperitoneum and pelvic lymph nodes is the last diagnosis which could be thought of for a 60 -year-old male patient presenting with dyspepsia and abdominal discomfort. A high degree of clinical suspicion is required to diagnose this condition. Retroperitoneal spread can occur with cancers of the pancreas, breast, cervix, endometrium, kidney, colon or prostate while pelvic lymphatic spread is generally from penile and testicular carcinomas. Our case is one such sporadic and unusual presentation where carcinoma of vallecula after definitive treatment with concurrent chemoradiotherapy (CCRT) metastasized directly to retroperitoneum and pelvis without involvement of its common metastatic sites with the primary disease under control.

\section{Case Presentation}

A 60-year-old male with no known comorbidities presented with history of progressive dysphagia and hoarseness of voice of four months duration. Patient was a chronic tobacco smoker for past 40 years. His hematological and biochemical parameters were within normal limits. Clinical evaluation revealed a $5 \times 4 \mathrm{~cm}$ ulcero-proliferative lesion involving right valleculla with no evidence of palpable neck nodes. Tissue biopsy from the lesion (Figure 1) revealed a moderately differentiated squamous cell carcinoma. Computed tomography (CT) scan of face and neck (Figure 2) showed a mass lesion measuring $2.4 \times 2.2 \times 3.4 \mathrm{~cm}$ epicentered in right vallecula involving base of tongue with multiple necrotic lymph nodes on ipsilateral side

Corresponding author: Dr Abhishek Purkayastha; Department of Radiation Oncology, Army Hospital Research and Referral, Dhaula Kuan, New Delhi, India.

Cite this article as: Purkayastha A, Sharma N, Suhag V. Extremely rare and unusual case of retroperitoneal and pelvic metastasis from squamous cell carcinoma of vallecula. Int J Cancer Ther Oncol. 2016; 4(2):4210. D0I: 10.14319/ijcto.42.10 
largest $1.4 \mathrm{~cm}$ right level II. Whole body positron emission tomography (WB PET) scan showed a $3 \times 3.2 \times$ $5 \mathrm{~cm}$ soft tissue thickening in oropharynx with multiple lymph nodes involving bilateral neck. Patient was staged as cT3N2cM0 Stage IVA. We treated him with definitive radiotherapy by 3 -dimensional conformal radiation therapy (3-DCRT) technique to a dose of 70 Gray (Gy) in 35 fractions along with concurrent chemotherapy inj cisplatin. Patient tolerated treatment well and was on regular follow up, but after six months, he presented with dyspepsia, abdominal discomfort and weight loss. An upper gastro-intestinal endoscopy (UGIE) was essentially normal. No evidence of any residual or recurrent disease was seen on local evaluation. WB PET scan showed complete metabolic resolution of primary lesion as well as pre-existing lymph nodes, however increased fluoro-deoxy-glucose (FDG) uptake was noted in retroperitoneal lymph nodes like porto-caval, pre-aortic, aorto-caval lymph nodes (Figure 3) and in right common iliac lymph nodes (Figure 4). CT guided FNAC from retroperitoneal and pelvic deposits revealed metastasis of squamous cell carcinoma (Figure 5). Patient was diagnosed as a case of carcinoma vallecula with retroperitoneal and pelvic metastasis and was managed with palliative chemotherapy inj paclitaxel, carboplatin and cetuximab. However, during treatment, his general condition deteriorated and chemotherapy was inadvertently stopped. He was thereafter kept on supportive care but finally succumbed to his metastatic disease.

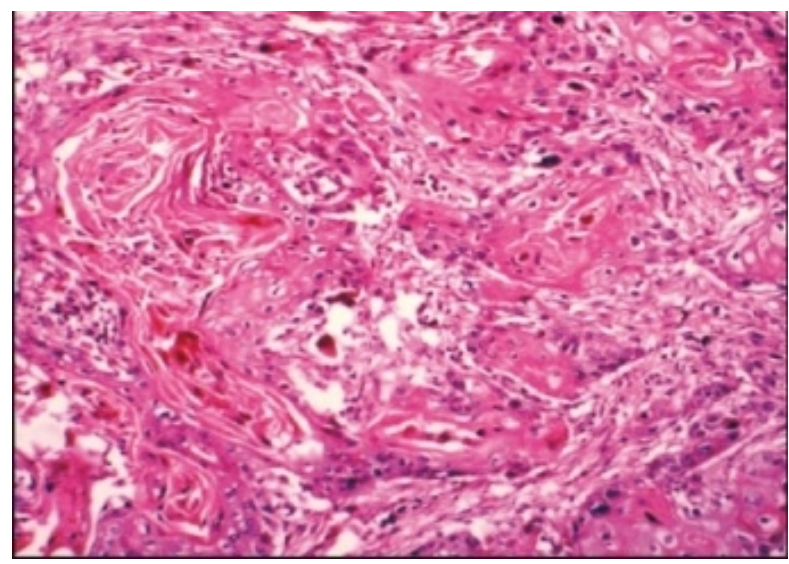

Figure 1: Tissue biopsy from vallecula showing moderately differentiated squamous cell carcinoma (H \& E $100 \mathrm{X}$ ).

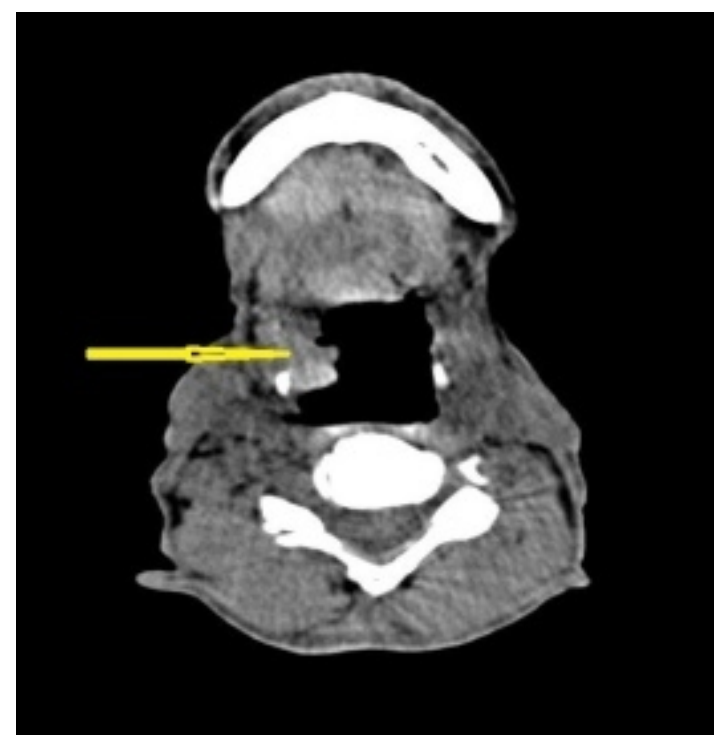

Figure 2: CT scan face and neck showing a mass lesion in right vallecula (yellow arrow).

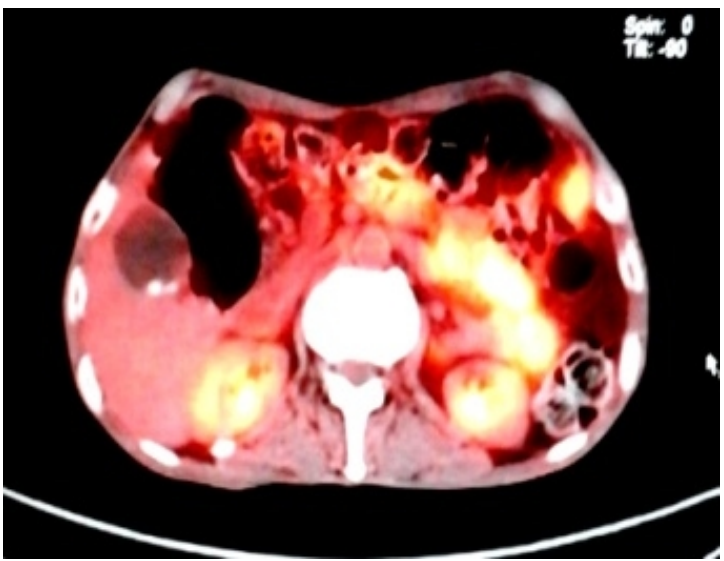

Figure 3: WB PET scan showing increased FDG uptake in retroperitoneal lymph nodes.

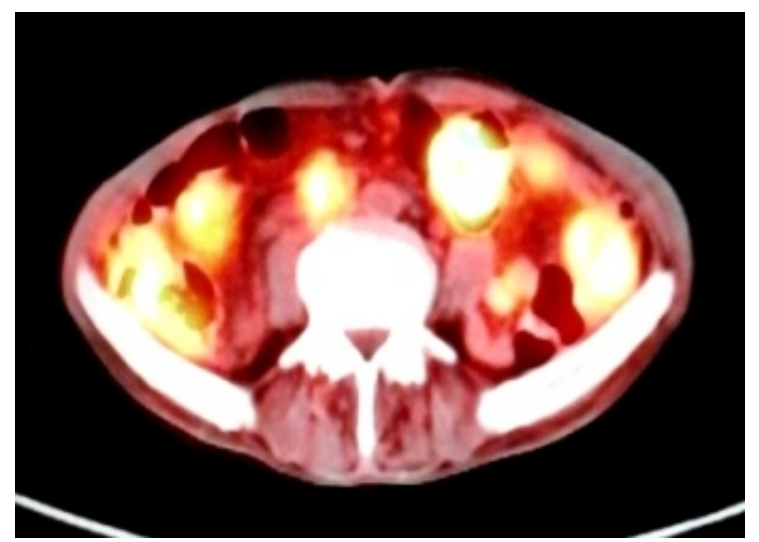

Figure 4: WB PET scan showing increased FDG uptake in right common iliac lymph nodes. 


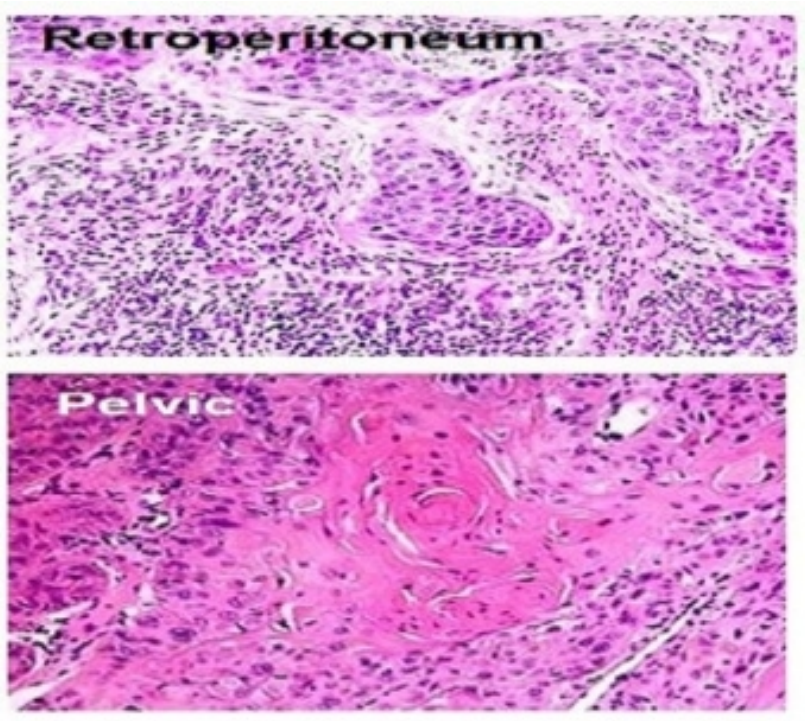

Figure 5: CT guided FNAC from retroperitoneal and pelvic deposits showing metastasis of squamous cell carcinoma (H \& E $40 \mathrm{X}$ ).

\section{Discussion}

The incidence of distant metastasis from head and neck carcinomas was less than one percent as was first reported in 1906 by Crile. ${ }^{3}$. However, there is a trend in rise of distant metastasis from head and neck malignancies in the present scenario of highly advanced diagnostic and curative modalities. It is probably because metastasis has become more evident in patients who survive longer than their usual life span. ${ }^{4}$ Distant metastasis in oropharyngeal carcinomas are more common in patients presenting with advanced locoregional and recurrent lesions, the risk increasing with tumor stage, size and lymphadenopathy i.e., N2 or N3 disease. ${ }^{1,5}$ Other important risk factors are involvement of level IV cervical lymph nodes, lymphovascular invasion and extranodal extension. ${ }^{6}$ Involvement of the retroperitoneum can occur most commonly by haematogenous dissemination while direct invasion, lymphatic spread and intraperitoneal seeding from exfoliated cancer cells are other modes of spread. ${ }^{7}$ Pelvic spread is mostly from lymphatic route. ${ }^{8}$ Patients with retroperitoneal metastasis usually remain asymptomatic. However, they may present with complaints of nausea, vomiting, abdominal discomfort, distension, pain, backache, frequency of urination, lymphedema or hydronephrosis. ${ }^{7}$ The most common sites of distant metastasis from oropharyngeal carcinoma are pulmonary parenchyma followed by bone and liver while metastasis to retroperitoneum and pelvic lymph nodes is extremely uncommon and has rarely been reported or written in standard oncology textbooks. ${ }^{1}$ Retroperitoneal metastasis generally have primaries from carcinoma ovary,,gastric carcinoma, colorectal carcinoma, carcinoma lung, peritoneal malignancy, carcinoma breast, testicular carcinomas and even frommalignant melanomas ${ }^{7,9}$ while common iliac lymph node metastasis have primaries from penis and testis $^{8}$ but not from head and neck malignancies. Differential diagnosis of retroperitoneal deposits can be peritoneal tuberculosis, peritoneal mesothelioma, benign calcifications or abdominal sepsis, but in all these cases, a primary tumor is not known.

Radiographic modalities like ultrasonography (USG), CT scan, magnetic resonance imaging (MRI) and PET scan help to distinguish these peritoneal and pelvic pathologies from metastatic lesion. CT scan can detect peritoneal metastasis in $85-93 \%$ cases $^{7}$ and they appear as soft tissue nodules, enhancement of peritoneal reflections, thickening of omentum or omental caking, distortion of mesentery, calcifications and ascites. MRI has proved to be more sensitive than CT scan in detection of metastatic lesions ${ }^{7}$ while PET imaging evaluates the biochemical and physiological characteristics of tumor cells, generating a radiographic picture of metabolic activity from the cancer nodule that is not possible with CT or MRI. These imaging techniques generally use lymph node size as the criteria for differentiating metastatic and benign lesions. Lymph nodes with a short-axis diameter more than $5 \mathrm{~mm}$ in the retroperitoneum and $6 \mathrm{~mm}$ in the pelvis are considered abnormal. ${ }^{9}$ The novel procedures of intraperitoneal contrast CT,10 lymphotropic nanoparticle enhanced MRI, ${ }^{8}$ diffusion-weighted $\mathrm{MRI}^{8}$ and $\mathrm{C}$-cholin PET scan ${ }^{8}$ are being used as a way to improve sensitivity of detection of retroperitoneal and pelvic metastasis. Regarding treatment, few studies in literature exist mainly from metastatic ovarian carcinomas and testicular carcinomas. Presently there are no definite guidelines for treatment of retroperitoneal or pelvic metastasis from carcinoma of oropharynx and the metastasis per se are not locally treated by surgery or radiotherapy, although systemic treatment with palliative chemotherapy may have some effect. While selecting therapy, overall survival should be taken into consideration. Complications like malignant ascites or bowel obstruction do however frequently require treatment for palliation. In our institute we used parenteral chemotherapeutic agents like paclitaxel, carboplatin and cetuximab a monoclonal antibody and an epidermal growth factor receptor (EGFR) inhibitor in our patient, though he was not able to complete the planned chemotherapy due to his deteriorating general condition and we were not able to establish its effect on overall survival of the patient.

\section{Conclusion}

The aim of reporting this case is to highlight its extreme rarity, mode of presentation, high degree of clinical suspicion required in detecting such an uncommon entity, multimodality treatment exhibited and the overall prognosis. The diagnosis of retroperitoneal metastasis should always be considered whenever any patient earlier treated for oropharyngeal carcinoma 
presents with vague abdominal discomfort or weight loss to enable a prompt initiation of appropriate treatment. Extensive retroperitoneal metastasis is known as peritoneal carcinomatosis which indicates a dismal prognosis and poor survival. Due to lack of any definitive management protocol and scarcity of relevant literature for retroperitoneal and pelvic metastasis from carcinoma of oropharynx, we recommend more studies, trials, case reports and researches on functional imaging modalities to be done in order to establish an acceptable treatment guideline for such disease.

\section{Conflict of interest}

The authors declare that they have no conflicts of interest. The authors alone are responsible for the content and writing of the paper.

\section{References}

1. Merino OR, Lindberg RD, Fletcher GH. An analysis of distant metastasis from squamous cell carcinoma of upper respiratory and digestive tracts. Cancer 1977; 40(1):145-51.

2. Hofmann U, O'Connor JP, Biyani CS, et al. Retroperitoneal metastatic squamous cell carcinoma of the tonsil (with elevated beta human chorionic gonadotrophin): a misdiagnosis as extra-gonadal germ cell tumour. J Laryngol Otol. 2006; 120(10): 885-7.

3. Crile GW. Carcinoma of the jaws, tongue, cheek and lips. Surg Gynecol Obstet 1923; 36:159-84.

4. Denington ML, Carter DR, Meyers AD. Distant metastasis in head and neck epidermoid carcinoma. Laryngoscope. 1980; 90:196-201.

5. Mcleod NM, Jess A, Anand R. Role of chest CT in staging of oropharyngeal cancer: a systematic review. Head Neck. 2009; 31(4): 548-55.

6. Goodwin WJ. Distant metastasis from oropharyngeal cancer. ORL. 2001; 63(4): 222-3.

7. Levy AD, Shaw JC, Sobin LH. Secondary tumors and tumorlike lesions of the peritoneal cavity: imaging features with pathologic correlation. Radiographics. 2009; 29 (2): 347-73.

8. Paño B, Sebastià C, Buñesch L, et al. Pathways of Lymphatic Spread in Male Urogenital Pelvic Malignancies. Radiographics. 2011; 31(1): 135-60.

9. Grubnic S, Vinnicombe SJ, Norman AR, et al. MR evaluation of normal retroperitoneal and pelvic lymph nodes. Clin Radiol. 2002; 57(3):193-200.

10. Halvorsen RA, Panushka C, Oakley GJ, et al. Intraperitoneal contrast material improves the CT detection of peritoneal metastases. AJR Am J Roentgenol. 1991;157 (1): 37-40. 